\title{
Rapid Detection of Multi-size Circular Shapes using Gradient Information and Signature Curve
}

\author{
Mahdi Abbasi \\ Department of Computer Engineering \\ Engineering Faculty, Bu-Ali Sina University, Hamedan, Iran
}

\begin{abstract}
The challenge of computational complexity reduction of Circular Hough Transforms (CHT) in detecting circular shapes in images is addressed. A new adaptive algorithm is introduced which minimizes the average accumulation space used in the voting process of CHT with the help of a variable size accumulation array. An improved method is presented which computes Signature Curve of gradient information of the image to find the radius and center of candidate circles, thus eliminating the influence of variable background intensity, especially in the noisy images. Experimental evaluations show that the proposed algorithm can significantly improve the quality of the results and considerably reduce the computational complexity and memory space.
\end{abstract}

\section{General Terms}

Computer Vision, Algorithm, circular Hough

\section{Keywords}

Hough Transform, Circle Detection, Gradient, Signature Curve

\section{INTRODUCTION}

The processing of an image to find items consisting of a line, circle and ellipse are considered as the significant issues of machine vision and image processing[1]. A lot of attention has been paid to finding circles in images which has a great application such as that in the industry to determine the location of circular items in images produced from the production line [2-10].

There have been offered various algorithms to recognize circles in the image, the best of which is the accumulation of the number of edges in forming the probable circle. Since the circle can be placed on each part of the image, usually in whatever size and direction, the set of all possibilities about the center and radius length of circle sample in any part of the image is considered. This method is called Signature Based Circular Hough Transform (SCHT) approach[1].

Despite the great popularity of SCHT lying in its simplified theory in implementing, this algorithm has a lot of backward. As recognizing the circle parametrically in an image requires three components $(\mathrm{x}, \mathrm{y}, \mathrm{r})$ in which $\mathrm{x}, \mathrm{y}$ are coordinates of circle center and $r$ the radius, the three-dimensional space of the algorithm is of abundant complexities of computation especially for noise-bearing co-centric circles[3, 11-13]. There have been offered various methods to escape these problems through separating parameter space $[1,14]$. Other methods tend to use randomly the image edge points along with geometrical features of circle to find a solution [15-19]. These methods, also, have weakness points $[1,2]$. For example, these restricted circulars Hough transforms have a low accuracy and speed especially in noise-bearing environments. Although these methods decreases the computation complexity, it has low efficiency due to performing search randomly on noise bearing images [20-24].In Probabilitybased Circular Hough Transform (PCHT) methods, only a proportion of image edge points (about 5 to 15\%) are used as representative points in storage increase followed by a search. Therefore PCHT methods reduce the computational complexity [15-19]. Although many accurate and efficient algorithms have been developed, none of them can control the number of false-positives, especially in image with co-centric or overlapping circles. The efficiency of these algorithms in low-noise environments is less than that in noise-bearing environments and even equal to that of SCHT algorithm. On the other hand, when these algorithms are used to search for co-centric circles with different radius, the problem of system memory shortage and computation time increase will arise due to using nested loops.

Given the lack of knowledge about the image, it is possible to find on efficient algorithm to find the location of circles in image. Using gradient direction of edge points to recognize the circle center is a basic and simple approach [20-22, 25]. The increasing need to memory in the above algorithms comes from using very great storage space for decision making about image edge points to find circle centers. Therefore, using small storage varying in the size suitable with connectivity area is an effective solution for this problem.

To find the circle radius and that of co-centric circles rapidly, it is possible to use signature curve method. That is, one can record the intensity changes in radius direction with in tolerance and expected range, instead of searching for all radii of circles, the center of which has been determined. The feature of this method is that the negative effect of the variable intensity of the image background in recognizing radius and circle periphery would vanish. The maximum points of the computation of signature curve values in radius range without using iterative loops will give us the correspondent radius with circle centers.

A new technique is presented, named Signature Based Circular Hough Transform (SBCHT) based on using uncertainty parameters in recognizing co-centric or noisebearing circles. The SBCHT extends Hugh transform and uses computation technique of edge point gradient field to recognize circle centers. It also uses signature curve to find correspondent radius with circle centers.

The paper is organized as follows. The next section focuses on using the combination of aforementioned techniques in rapid and accurate detection of circles. Algorithm implementation and experimental results are explained in section 3. Section 4 is allocated to results and conclusions.

\section{PROPOSED METHOD}

Figure 1 shows a flowchart representing used processing strategy as the main idea in the algorithm of recognizing samples of circular objects in the image. This includes the increase of the small storage of Hugh parameter space and 
intelligent application of this information to improve the parameter range so that more and better details are extracted from areas of interest. The determined parameters are used by inverse mapping to recognize the points not existing in the structure of the figure in question.

Other processing to assure the validity of determined points from the circular object is necessary. If these points obtain the required validity, they are removed from the initial list and the search is done with less resolution to find other samples of the object in the image. Therefore the algorithm repetition will continues until the parameter space gets an important structure in the images with various samples of object. The most important decision in this harmonic method is to determine the size of parameter space storage. In any case, optimal using of small storage of parameter space is such that the total connectivity of parameter space is maintained. As soon as the storage calculation is completed, Hugh parameter space is examined to find the maximum of the parameters. The procedures of maximal detection can be performed by using an algorithm which makes a binary array corresponding to the storage, labels, and examines the connected parts in the binary array. The algorithm then transforms the connected parts of the binary array in to a symbolic array in which cells of zero content remain the same and there is assigned a distinctive number to any subset of one.

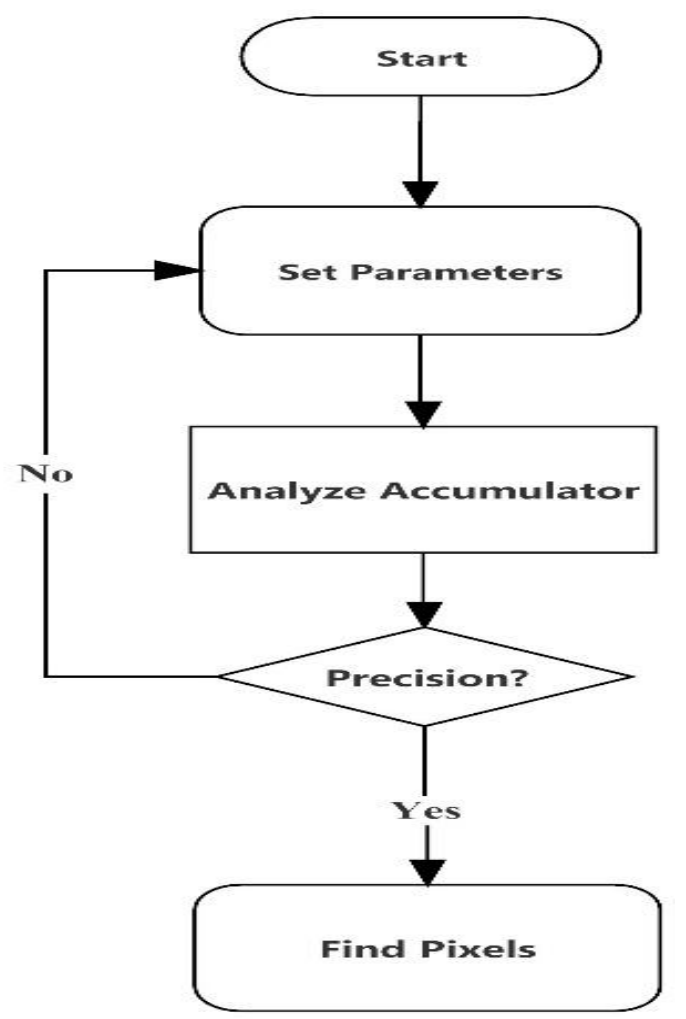

Figure 1: SBCHT flowchart

Labelling is done with the rectangular scan of image Repeating the algorithm on the image can help us to decide about the equivalent labels. The smallness of storage array and the fact that the search is done with one repetition shows that the computational burden and the required memory are both miniscule. In the proposed algorithm, the density of interconnected parts is a condition used to recognize the best maximal peak. That is, the calculated value in storage cells can be a criterion in recognizing the peak. Decision making about the restriction on the next iteration parameters of the algorithm is largely dependent on shape, spread and the place of the best available peak.

The best and most important aspect of SBCHT algorithm is its ability in redefinition of parameters independently in each iteration. The center of new parameter range is located in the center of the best peaks. The width of new range is the same as the connective section in each of parameter aces. The amount of used memory in this approach is much less than that in the standard SCHT. As mentioned, reducing the size of parameter space storage assures the advantages of this algorithm.

\subsection{Gradient of edge points}

The first technique used to find the circles rapidly is to apply the concept of illumination intensity gradient of image edge points. Having applied threshold algorithms and found the connected parts, the gradient field of illumination intensity in each AOI (Area of Interest) by using the following equation while the image connectivity is determined end areas of interest and probability are recognized:

$$
\begin{aligned}
& \Delta I(i, j)=\left.\left(v_{x}, v_{y}\right)\right|_{(i, j)}= \\
& (I(i, j)-I(i, j-1), I(i, j)-I(i-1, j))
\end{aligned}
$$

In this equation, $(i, j)$ are pixel indices, $\Delta I(i, j)$ the gradient vector of pixel $(i, j)$ which has some parts in $\mathrm{x}, \mathrm{y}$ direction and, $\mathrm{I}(i, j)$ is illumination intensity of the image. The intersections of all nonzero gradient vectors in the points belonging to a disc or a circle (developed shape of disc) refer to the center of disc or circle. Therefore, applying a simple transform based on the gradient field of each image point and then forming a storage which holds the intensity of the pixel is proportional with this probability that the mentioned pixel is the circle center. The intensity maximum in mapping space storage shows the most probable center. Therefore, the storage array had better have the dimension of gradient fiend. Storage array is formed in a voting procedure. Figure 2 shows the related process. There is added a weight value to pixels of storage array in the direction of $\nabla(i, j)$ for each nonzero gradient of $\nabla(i, j)$ in gradient field. Length of this vector is equal to the maximum of specified radius for the circle and the value of gradient amplitude $\nabla(i, j)$ is chosen as its weight value. Having collected the votes about all nonzero gradient vectors, the most valuable point in storage is chosen (figure 2.b) as the circle center. For every established AOI in an image with several discs and circles, there will be some please for storage array corresponding with the AOI. Recognizing the local maxima in the storage array is as follows:

1. A filter to find the local maxima is applied to storage array. The filter fills the passage area between local maxima with zero or negative value. This facilitates the distinction of local maxima in the next stages.

2. Thresholding storage array is performed by choosing a positive value as a threshold. Thresholding causes local maxima and other points to be specified with label one and zero, respectively. Then, the areas with local maxima are distinguished by an algorithm of region growing and weighted mass center of each is considered as the location of local maximum in that area. 


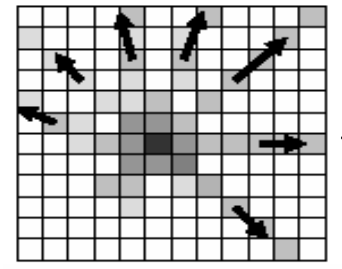

(a)

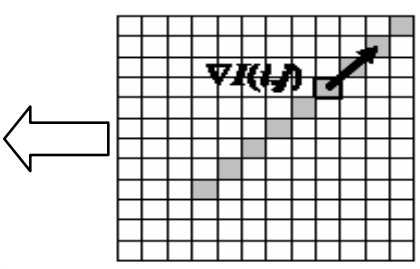

(b)
Figure 2: Construction of accumulator

a) Accumulator before voting

b) Accumulator after voting

Every local maximum in storage array is corresponding to the center of a circle or a disc in the image. Having calculated the location of storage local maxima, one can obtain the real location of circle centers on image by inverse mapping.

Suppose that $\left(i_{c}, j_{c}\right)$ denotes the circle center in question, vector $\overrightarrow{V(l, j)}$ shows the gradient vector for a pixel $(i, j)$ in the neighbourhood of $\left(i_{c}, j_{c}\right)$. If $\overrightarrow{q(l, j)}$ is a vector from the center to pixel $(i, j)$, the function $f(r)$, as the calculator of signature carve value, is obtained by formula (2).

$$
f(r)=\frac{\sum_{|q \rightarrow(i, j)-r|<\Delta r / 2}[\stackrel{\mu}{V}(i, j) \cdot \stackrel{\rho}{\rho}(i, j) /|q(i, j)|]}{\sum_{|q \rightarrow(i, j)-r|<\Delta r / 2}^{\rho} 1}
$$

For each $r$ values in pre-specified range of radius, the summation regarding center $\left(i_{c}, j_{c}\right)$ is computed. The signature curve records the changes of illumination intensity in radius direction for the circle radius in the defined range. The negative effect of variable intensity on image background to recognize the radius accurately is removed by using this method. In fact, algorithm uses the symmetry feature of circle and calculates the curve value in each radius $r$ as the mean in tangent direction. It is definitely proved that the local maxima in this summation show the circle radius of center $\left(i_{c}, j_{c}\right)$.

Therefore, using another storage, with the dimension the some as probable radius maximum, it is found that $f(r)$ for each circle centers $\left(i_{c}, j_{c}\right)$ specified in searching phase of centers. A smoothing filter can be used to delete the small noise of the storage to find local maximum point. Having ordered them ascending, it can be found the distance between each pair of them. The passage distance between these local maxima shows the validity or non-validity of found radii. The input parameter of the algorithm for the certainty criterion in choosing adjacent discs helps experimentally to decide intelligently in multi-radius case.

\section{EXPERIMENTAL RESULTS}

A bank of 100 images of $256 \times 256$ was used to test SBCHT method. Each image had one or more circle with various radii and different locations. There were found some imperfect circles in images. Figure 3 shows two samples of these images along with the result of implementing SBCHT algorithm.

As seen from figure 3, algorithm efficiency is acceptable even in the presence of noise. In image (b) with higher noise then image (a) before addition of salt \& pepper noise, the algorithm could recognize the circle correctly by only reducing gradient threshold. In assessing the computational complexity of the algorithm and comparing it with that of the standard SCHT, the counting of voting applications on each algorithm for 50 images of $256 \times 256$ was used and then calculated the mean of this criterion as a computational complexity criterion, based on idea proposed in [16, 20-23, 26]. The corresponding results of Table 1 show that in all cases the SBCHT is faster than SCHT.

The result of this rapid search is the favorite multi-circular and multi radial objects of the image. Figure 4 shows the results of a non-repetitive execution of SCHT and SBCHT algorithms on a sample image. In this test, SBCHT can find all the available circles in 2.47 seconds while it takes 121.99 second for SCHT to find only the circle with the biggest radius. It is clear that five other repetition of SCHT is needed to find other five circles.

Another test was used to examine the strength of SBCHT with noise. The error of SCHT and SBCHT were investigated in this test by increasing the noise of salt $\&$ pepper from $0 \%$ to $100 \%$. Figure 5 shows the error curve of two algorithms with the increase of noise density.

The plots of figure 5 show that the presence of noise to $40 \%$ doesn't change the accuracy of SBCHT algorithm while SCHT loses its accuracy with a noise of $10 \%$ density. The SCHT fails completely by adding a noise of $50 \%$ density while the SBCHT tolerates the noise of $70 \%$ density.
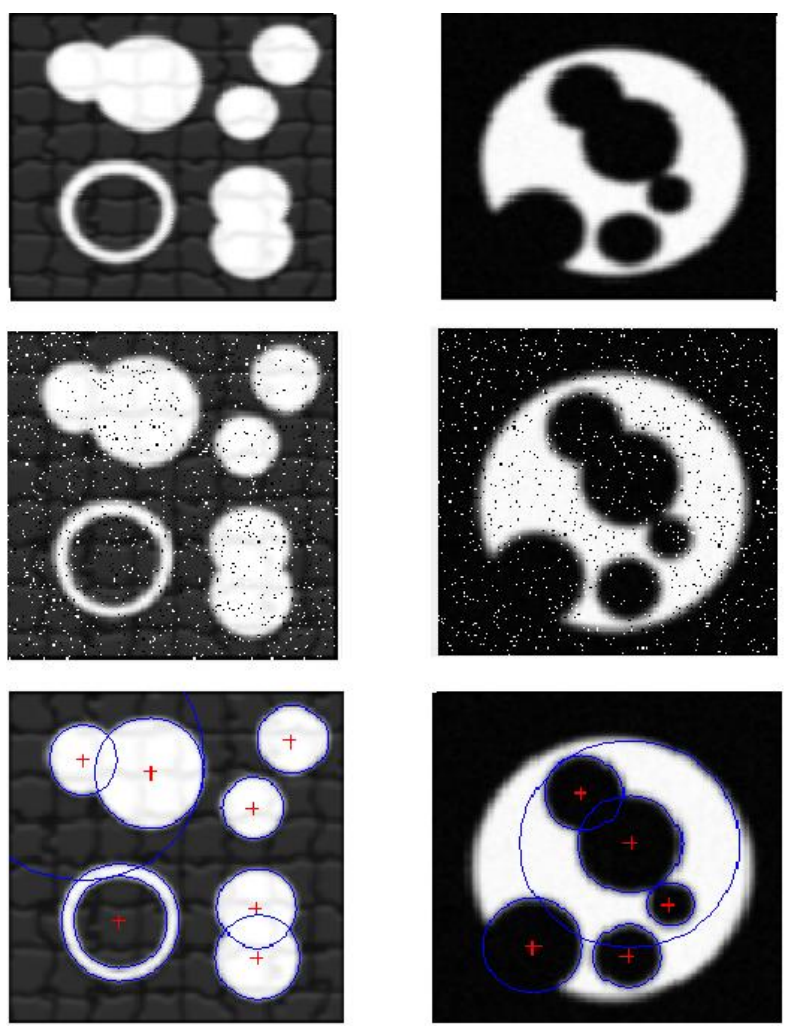

Figure 3: Result of applying SBCHT on images From Top: initial image, Noisy image (pepper noise 0.05 ), and Detected Circles. 
Table 1: Comparing average voting times of SBCHT and SCHT

\begin{tabular}{|c|c|c|c|}
\hline & $\begin{array}{c}\text { Image } \\
\text { without } \\
\text { noise }\end{array}$ & $\begin{array}{c}\text { Image with } \\
\text { Salt Pepper } \\
\text { noise } \\
(0.10)\end{array}$ & $\begin{array}{c}\begin{array}{c}\text { Image } \\
\text { with }\end{array} \\
\text { Gaussian } \\
\text { noise }\end{array}$ \\
\hline $\begin{array}{l}\text { Voting in } \\
\text { SCHT }\end{array}$ & 65536 & 68234 & 67552 \\
\hline $\begin{array}{l}\text { Voting in } \\
\text { SBCHT }\end{array}$ & 14133 & 15123 & 15221 \\
\hline $\begin{array}{c}\text { Average } \\
\text { Voting time } \\
\text { in } \\
\text { SCHT }\end{array}$ & 205 & 319 & 262 \\
\hline $\begin{array}{c}\text { Average } \\
\text { Voting time } \\
\text { in } \\
\text { SBCHT }\end{array}$ & 2.10 & 4.36 & 2.92 \\
\hline
\end{tabular}

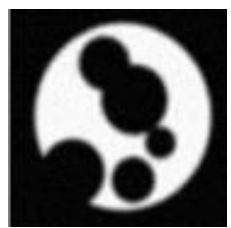

(a)

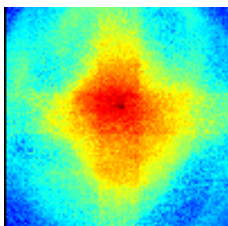

(b)

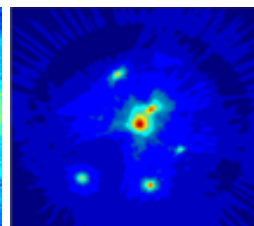

(c)
Fig 4: Multi radius function of SBCHT a) Sample image b) Hough Space of SCHT c) Hough Space of SBCHT

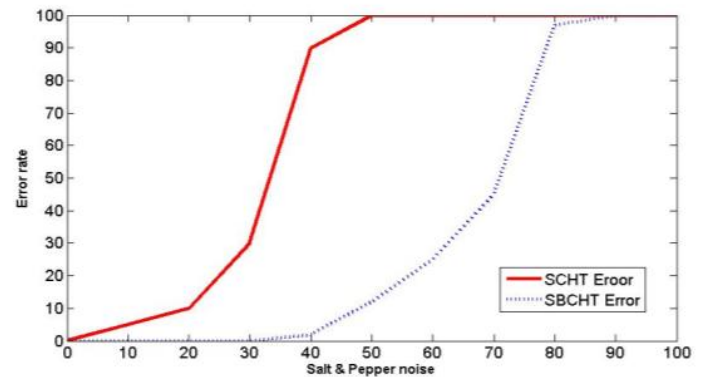

Figure 5. Comparing the robustness of SBCHT and SCHT

\section{CONCLUSION}

A new algorithm, SBCHT, was presented in this article to find circles brighter or darker than the environment independent from radius and perfectness of the shape, in which gradient and signature curve are the central techniques. There are various applications of SBCHT based on its speed and accuracy, among which are investigating MRI in medicine, face detection and microscopic applications in Nano environment. The results showed that SBCHT performs about 100 times as fast as SCHT algorithm and tolerates to $70 \%$ noise due to its high sustainability. The proposed method can help to recognize co-centric circles and co-centric disc and circles.

The feature work in optimizing this algorithm is to use statistical methods to estimate gradient threshold parameters by the algorithm so that the speed and accuracy of the algorithm would increase.

\section{REFERENCES}

[1] P. Mukhopadhyay and B. B. Chaudhuri, "A survey of Hough Transform," Pattern Recognition, vol. 48, pp. 993-1010, 2015/03/01/ 2015.

[2] J. Illingworth and J. Kittler, "A survey of the hough transform," Computer Vision, Graphics, and Image Processing, vol. 44, pp. 87-116, 1988/10/01/ 1988.

[3] T. D'Orazio, C. Guaragnella, M. Leo, and A. Distante, "A new algorithm for ball recognition using circle Hough transform and neural classifier," Pattern Recognition, vol. 37, pp. 393-408, 2004/03/01/ 2004.

[4] M. Y. Cao, C. H. Ye, O. Doessel, and C. Liu, "Spherical parameter detection based on hierarchical Hough transform," Pattern Recognition Letters, vol. 27, pp. 980986, 2006/07/01/ 2006.

[5] R. Varun, Y. V. Kini, K. Manikantan, and S. Ramachandran, "Face Recognition Using Hough Transform Based Feature Extraction," Procedia Computer Science, vol. 46, pp. 1491-1500, 2015/01/01/ 2015.

[6] Y. Wang and G. Cheng, "Application of gradient-based Hough transform to the detection of corrosion pits in optical images," Applied Surface Science, vol. 366, pp. 9-18, 2016/03/15/ 2016.

[7] M. Sengupta and J. K. Mandal, "Authentication Through Hough Transformation Generated Signature on G-Let D3 Domain (AHSG)," Procedia Technology, vol. 10, pp. 121-130, 2013/01/01/ 2013.

[8] A. A. Kassim, Z. Mian, and M. A. Mannan, "Connectivity oriented fast Hough transform for tool wear monitoring," Pattern Recognition, vol. 37, pp. 1925-1933, 2004/09/01/ 2004.

[9] J. Cha, R. H. Cofer, and S. P. Kozaitis, "Extended Hough transform for linear feature detection," Pattern Recognition, vol. 39, pp. 1034-1043, 2006/06/01/ 2006.

[10] E. A. Murillo-Bracamontes, M. E. Martinez-Rosas, M. M. Miranda-Velasco, H. L. Martinez-Reyes, J. R. Martinez-Sandoval, and H. Cervantes-de-Avila, "Implementation of Hough transform for fruit image segmentation," Procedia Engineering, vol. 35, pp. 230239, 2012/01/01/ 2012.

[11] M.-L. Torrente, S. Biasotti, and B. Falcidieno, "Recognition of feature curves on 3D shapes using an algebraic approach to Hough transforms," Pattern Recognition, vol. 73, pp. 111-130, 2018/01/01/2018.

[12] M. C. Beltrametti and L. Robbiano, "An algebraic approach to Hough transforms," Journal of Algebra, vol. 371, pp. 669-681, 2012/12/01/2012.

[13] M. Bernal-Marin and E. Bayro-Corrochano, "Integration of Hough Transform of lines and planes in the framework of conformal geometric algebra for 2D and 3D robot vision," Pattern Recognition Letters, vol. 32, 
pp. 2213-2223, 2011/12/01/ 2011.

[14] Tsuji and Matsumoto, "Detection of Ellipses by a Modified Hough Transformation," IEEE Transactions on Computers, vol. C-27, pp. 777-781, 1978.

[15] N. Kiryati, H. Kälviäinen, and S. Alaoutinen, "Randomized or probabilistic Hough transform: unified performance evaluation," Pattern Recognition Letters, vol. 21, pp. 1157-1164, 2000/12/01/ 2000.

[16] C. Galamhos, J. Matas, and J. Kittler, "Progressive probabilistic Hough transform for line detection," in Proceedings. 1999 IEEE Computer Society Conference on Computer Vision and Pattern Recognition (Cat. No PR00149), 1999, pp. 554-560 Vol. 1.

[17] H. Kälviäinen, P. Hirvonen, L. Xu, and E. Oja, "Probabilistic and non-probabilistic Hough transforms: overview and comparisons," Image and Vision Computing, vol. 13, pp. 239-252, 1995/05/01/ 1995.

[18] A. Yla-Jaaski and N. Kiryati, "Adaptive termination of voting in the probabilistic circular Hough transform," IEEE Transactions on Pattern Analysis and Machine Intelligence, vol. 16, pp. 911-915, 1994.

[19] N. Kiryati, Y. Eldar, and A. M. Bruckstein, "A probabilistic Hough transform," Pattern Recognition, vol. 24, pp. 303-316, 1991/01/01/ 1991.

[20] A. O. Djekoune, K. Messaoudi, and K. Amara,
"Incremental circle hough transform: An improved method for circle detection," Optik, vol. 133, pp. 17-31, 2017/03/01/ 2017.

[21] Z. Yao and W. Yi, "Curvature aided Hough transform for circle detection," Expert Systems with Applications, vol. 51, pp. 26-33, 2016/06/01/ 2016.

[22] M. Ujaldón, A. Ruiz, and N. Guil, "On the computation of the Circle Hough Transform by a GPU rasterizer," Pattern Recognition Letters, vol. 29, pp. 309-318, 2008/02/01/ 2008.

[23] D. Ioannou, W. Huda, and A. F. Laine, "Circle recognition through a $2 \mathrm{D}$ Hough Transform and radius histogramming," Image and Vision Computing, vol. 17, pp. 15-26, 1999/01/01/ 1999.

[24] H. K. Yuen, J. Princen, J. Illingworth, and J. Kittler, "Comparative study of Hough Transform methods for circle finding," Image and Vision Computing, vol. 8, pp. 71-77, 1990/02/01/ 1990.

[25] H. Yang, J. Luo, Z. Shen, and W. Wu, "A local voting and refinement method for circle detection," Optik, vol. 125, pp. 1234-1239, 2014/02/01/ 2014.

[26] C. Kimme, D. Ballard, and J. Sklansky, "Finding circles by an array of accumulators," Commun. ACM, vol. 18, pp. 120-122, 1975. 